\title{
Interaction between proatherosclerotic factors and right-to-left shunt on the risk of cryptogenic stroke: the Italian Project on Stroke in Young Adults
}

\author{
Alessandro Pezzini, ${ }^{1}$ Mario Grassi, ${ }^{2}$ Corrado Lodigiani, ${ }^{3}$ Rosalba Patella, ${ }^{4}$ Carlo Gandolfo, ${ }^{5}$ \\ Andrea Zini, ${ }^{6}$ Rossella Musolino, ${ }^{7}$ Rocco Salvatore Calabrò, ${ }^{8}$ Paolo Bovi, ${ }^{9}$ \\ Alessandro Adami, ${ }^{10}$ Maria Luisa DeLodovici, ${ }^{11}$ Elisabetta Del Zotto, ${ }^{1}$ \\ Lidia Luciana Rota, ${ }^{3}$ Maurizia Rasura, ${ }^{4}$ Massimo Del Sette, ${ }^{12}$ Alessandra Spalloni, \\ Alessia Giossi, ${ }^{1}$ Irene Volonghi, ${ }^{1}$ Federica Casoni, ${ }^{6}$ Paolo Cerrato, ${ }^{13}$ Paolo Costa, ${ }^{1}$ \\ Mauro Magoni, ${ }^{14}$ Antonella Toriello, ${ }^{15}$ Maurizio Paciaroni, ${ }^{16}$ Giorgio Dalla Volta, ${ }^{17}$ \\ Licia lacoviello, ${ }^{18}$ Alessandro Padovani, ${ }^{1}$ on behalf of the Italian Project on Stroke in \\ Young Adults (IPSYS) Investigators
}

\begin{abstract}
- An additional appendix is published online only. To view this file please visit the journal online (http://heart.bmi.com/ content $/ 98 / 6 . t o c$ )
\end{abstract}

For numbered affiliations see end of article.

\section{Correspondence to} Dr Alessandro Pezzini, Dipartimento di Scienze Mediche e Chirurgiche, Clinica Neurologica, Università degli Studi di Brescia, P.le Spedali Civili, 1, 25100 Brescia, Italia; ale pezzini@hotmail.com

Accepted 5 January 2012 Published Online First 23 January 2012

\begin{abstract}
Objective To explore the interaction effects between cardiac interatrial right-to-left shunt (RLS) and proatherosclerotic factors on the risk of brain ischaemia.

Design Multicentre Italian case-control study.

Setting University hospitals.

Participants 588 patients with cryptogenic stroke (CS) aged $\leq 45$ years and 585 control subjects consecutively enrolled as part of the Italian Project on Stroke in Young Adults.
\end{abstract}

Methods Interaction effects between RLS and an individual proatherosclerotic score computed from the number of conventional vascular risk factors for the risk of CS were investigated. Data were examined by logistic regression models and expressed as interaction $\mathrm{OR}$ or interaction risk difference (RD).

Results CS risk increased with increasing number of proatherosclerotic factors in subjects without RLS IOR 2.73; $95 \% \mathrm{Cl} 1.98$ to $3.76 ; \mathrm{RD}+0.246 ; 95 \% \mathrm{Cl}+0.17$ to +0.32 ; for subjects with one or more factors), but was higher in subjects with RLS and no additional proatherosclerotic factors (OR $5.14 ; 95 \% \mathrm{Cl} 3.49$ to 7.58 ; $\mathrm{RD}+0.388 ; 95 \% \mathrm{Cl}+0.31$ to +0.47 ) compared with subjects without RLS and no risk factors. Negative interaction and antagonistic effects between RLS and proatherosclerotic factors were observed (interaction $\mathrm{OR}$ 0.52; 95\% Cl 0.31 to 0.91; interaction $\mathrm{RD}-0.17 ; 95 \% \mathrm{Cl}$ -0.29 to -0.05$)$.

Conclusions The influence of RLS on the risk of CS decreases with increasing number of atherosclerotic factors, and is highest when such factors are absent. Individual proatherosclerotic profiles may help to identify patients with CS whose patent foramen ovale is probably pathogenic.

\section{INTRODUCTION}

The role of patent foramen ovale (PFO) as a risk factor for stroke is controversial. Although the numerous case-control studies conducted thus far have demonstrated an increased prevalence of this cardiac abnormality and interatrial right-to-left shunt (RLS) in patients with cryptogenic stroke (CS) compared with patients with stroke of known cause, ${ }^{1}$ two population-based prospective studies did not confirm these findings, ${ }^{2}{ }^{3}$ questioning the concept of increased stroke risk from this anatomical variant. Apart from the obvious differences in the design of the studies, one likely reason for these inconsistencies is the substantial heterogeneity of patients with RLS and otherwise unexplained stroke, as a consequence of the influence that additional cofactors might have on this relation. Actually, numerous factors can potentially affect the degree of association and thus the likelihood that a PFO in the setting of CS is an incidental finding, leading us to assume that only specific subgroups of PFO carriers are exposed to a relevant stroke risk. Identifying patients whose stroke is probably attributable to PFO may be useful in riskpredictive modelling aimed at reducing treatment effect heterogeneity and, on an individual level, in selecting the most appropriate option for secondary prevention. ${ }^{4}$ In this regard, sparse reports have suggested, although not always in a consistent direction, that RLS is associated with CS when patients are younger and have a lower prevalence of conventional atherosclerotic risk factors. ${ }^{5-10}$ However, since none of these analyses have included a control group composed of stroke-free subjects whose PFO status had been determined, any estimation about how the risk of stroke in PFO carriers may vary depending on the presence or absence and burden of such cofactors is precluded.

The purpose of the present study is to provide such an estimation based on a case-control analysis including a large, homogeneous and wellcharacterised patient population from the Italian Project on Stroke in Young Adults (IPSYS). In particular, we sought to explore any interaction effect between RLS and atherosclerotic cofactors on CS risk. 


\section{SUBJECTS AND METHODS}

The study was approved by the local ethics committee. Informed consent was provided by all study participants.

\section{Study group \\ Cases}

Stroke patients were recruited in the setting of the IPSYS. IPSYS is a countrywide network of neurological centres with special interest in cerebral ischaemia at a young age across Italy (see online appendix), aimed at recruiting patients with first-ever acute ischaemic stroke, who fulfil the criteria age 18-45 years and CT- or MRI-proven cerebral infarction, in the setting of a hospital-based, multicentre, observational study. ${ }^{11}$ Centres are included in the network provided that the recruitment process of stroke cases takes place prospectively. Stroke was defined as a sudden loss of global or focal cerebral function that persisted for $>24 \mathrm{~h}$ with a probable vascular cause. Ischaemic strokes due to sinus venous thrombosis, vasospasm after subarachnoid haemorrhage or cardiac surgery, or occurring as an immediate consequence of trauma, and iatrogenic strokes were excluded. For the purpose of the present analysis, we screened datasets from patients included between January 2000 and July 2009.

\section{Clinical and laboratory investigations}

All patients underwent an extensive aetiological workup aimed at determining the most likely mechanism of stroke in each case; it included complete blood cell count, biochemical profile, urinalysis, 12-lead ECG, chest roentgenography, Doppler ultrasonography with frequency spectral analysis and B-mode echotomography of the cervical arteries, transcranial Doppler ultrasonography, and CT and/or MR angiography to investigate extracranial and intracranial vessels. The performance of specialised coagulation testing (including prothrombin and activated partial thromboplastin times, antibodies to phospholipid, fibrinogen, protein $\mathrm{C}$, protein $\mathrm{S}$, activated protein $\mathrm{C}$ resistance, antithrombin III, genotyping to detect factor $\mathrm{V}$ Leiden and the G20210A mutation in the prothrombin gene) was left to the discretion of the investigator in charge of the patient. Transthoracic and/or transoesophageal echocardiography was performed to rule out cardiac sources of emboli. In particular, interatrial RLS was assessed in all patients by transoesophageal echocardiography with a contrast study and Valsalva manoeuvre (c-TEE) and/or transcranial Doppler sonography with intravenous injection of agitated saline (c-TCD). An RLS was considered present if any microbubble was seen in the left atrium within three cardiac cycles from maximum right atrial opacification on echocardiography. ${ }^{12}$ c-TCD was performed according to the Venice Consensus Conference. ${ }^{13}$ Briefly, it consists of the injection of $9 \mathrm{ml}$ saline solution and $1 \mathrm{ml}$ air mixed with a threeway stopcock by exchange of saline/air mixture between the syringes and injected as a bolus as a contrast-enhancing agent into the right cubital vein $5 \mathrm{~s}$ before the start of a $10 \mathrm{~s}$ Valsalva manoeuvre, while recording the flow velocity of the middle cerebral artery, insonated through the temporal window on the right side at a depth of $50-60 \mathrm{~mm}$, with a handheld probe. The appearance of transient spikes on the velocity spectral curve is considered positive for interatrial RLS. The method has an overall diagnostic accuracy comparable to that of c-TEE. ${ }^{14}$ Patients were categorised according to an aetiological classification based on the Trial of Org 10172 in Acute Stroke Treatment criteria, accommodated and validated for stroke in the young. ${ }^{15}$ Definite causes of stroke included the following aetiological categories: (1) atherosclerotic vasculopathy, (2) non-atherosclerotic vasculopathy, (3) small-vessel disease, (4) probable cardi- oembolism, (5) haematological cause (coagulopathies), and (5) migrainous stroke. CS was defined as cerebral infarcts that did not meet the criteria for one of the categories mentioned above and fulfilled the diagnostic criteria for the categories (1) cardiac/ transcardiac embolism with RLS only, (2) use of oral contraceptive or exogenous oestrogen and (3) indeterminate. ${ }^{15}$

\section{Risk factor definition}

The following risk factors for premature cerebral ischaemia were retained: hypertension, diabetes mellitus, cigarette smoking and hypercholesterolaemia. These variables were defined and dichotomised as follows: hypertension (systolic blood pressure $\geq 140 \mathrm{~mm} \mathrm{Hg}$ and diastolic blood pressure $\geq 90 \mathrm{~mm} \mathrm{Hg}$ in two separate measurements after the acute phase or use of antihypertensive drugs before recruitment), diabetes mellitus (history of diabetes, use of hypoglycaemic agent or insulin, or fasting glucose $\geq 126 \mathrm{mg} / \mathrm{dl}$ ), current smoking (including former smokers who had quit smoking for 6 months before the index event) and hypercholesterolaemia (serum cholesterol levels $\geq 220 \mathrm{mg} / \mathrm{dl}$ or use of cholesterol-lowering drugs).

\section{Control subjects}

Staff members of participating hospitals, with no known history of vascular disease, aged $\leq 45$ years, and from the same ethnic background as the cases were invited to participate in the study as controls. Demographic variables, vascular risk factors, and the presence of RLS on c-TCD were assessed in all these subjects according to the diagnostic criteria applied to the cases. ${ }^{11}$

\section{Statistical analysis}

On the basis of the number of the above mentioned cardiovascular risk factors, we computed an individual proatherosclerotic score (PS, from 0 to 4 ) and defined a binary variable (0 vs 1 or more). Descriptive differences among study groups were examined with the $\chi^{2}$ test, and by analysis of variance $F$ test, when appropriate. Logistic regression models were planned to examine the conditional ('pure') effects and any interaction (multiplicative or additive) effect of RLS and PS on the disease outcome, adjusted for age and gender. The interaction effect defines the situation where the effect of RLS on CS is different across strata of PS, or, vice versa, the effect of PS on CS is different across congenital anomaly groups. Multiplicative interaction occurs when the combined effect is larger (positive interaction) or smaller (negative interaction) than the product of the pure

Table 1 Demographic characteristics of patients with cryptogenic stroke and control subjects

\begin{tabular}{lccr}
\hline Characteristic & $\begin{array}{l}\text { Cryptogenic stroke } \\
(\mathbf{n = 5 8 8})\end{array}$ & $\begin{array}{l}\text { Control Subject } \\
(\mathbf{n = 5 8 5 )}\end{array}$ & $\mathbf{p}$ Value \\
\hline Age (years), mean \pm SD & $35.3 \pm 7.5$ & $34.0 \pm 8.0$ & 0.003 \\
Sex, women & $302(51.4)$ & $310(53.0)$ & 0.576 \\
Hypertension & $110(18.7)$ & $27(4.6)$ & $<0.001$ \\
Diabetes mellitus & $16(2.7)$ & $14(2.4)$ & 0.722 \\
Current smokers & $216(36.7)$ & $152(26.0)$ & $<0.001$ \\
Hypercholesterolaemia & $141(24.1)$ & $68(11.6)$ & $<0.001$ \\
Proatherosclerotic score & & & $<0.001$ \\
0 & $242(41.3)$ & $361(61.7)$ & \\
1 & $235(40.1)$ & $190(32.5)$ & \\
2 & $82(14.0)$ & $31(5.3)$ & \\
3 & $25(4.3)$ & $3(0.5)$ & \\
$\quad 4$ & $2(0.3)$ & $0(0.0)$ & \\
Right-to-left shunt & $279(47.5)$ & $119(20.3)$ & $<0.001$ \\
\hline
\end{tabular}

Values are number (\%) unless otherwise stated. 
Table 2 Right-to-left shunt and proatherosclerotic score interaction effect on the risk of cryptogenic stroke

\begin{tabular}{llllll}
\hline $\begin{array}{l}\text { Right-to-left } \\
\text { shunt }\end{array}$ & Proatherosclerotic score & Cases* $^{*}$ & Controls & OR (95\% CI) & RD (95\% Cl) $\dagger$ \\
\hline Absent & 0 & $114(19.8)$ & $303(51.8)$ & 1 & 0 \\
& 1 or more & $181(31.5)$ & $163(27.9)$ & $2.73(1.98$ to 3.76$)$ & $+0.246(+0.17$ to +0.32$)$ \\
Present & 0 & $125(21.8)$ & $62(10.6)$ & $5.14(3.49$ to 7.58$)$ & $+0.388(+0.31$ to +0.47$)$ \\
& 1 or more & $154(26.9)$ & $57(9.7)$ & $7.38(4.97$ to 11.0$)$ & $+0.462(+0.38$ to +0.54$)$ \\
\hline
\end{tabular}

Values for cases and controls are number (\%).

*14 cases had missing values.

†RDs were computed using the maximum value of Manski bounds on the risk difference in case-control studies. ${ }^{17}$

Interaction OR $(95 \% \mathrm{Cl})=7.38 /(2.73 \times 5.14)=0.526(0.31$ to 0.91$)$.

Interaction risk difference $(95 \% \mathrm{Cl})=0.46-(0.24+0.38)=-0.17(-0.29$ to -0.05$)$.

$\mathrm{RD}$, risk difference.

individual effects. Additive interaction occurs when the combined effect is larger (synergism) or smaller (antagonism) than the sum of the pure individual effects. The degree of interaction as a departure from multiplicative or additive scales, measured by the interaction $\mathrm{OR}$ or interaction risk difference $(\mathrm{RD})$ indices, was derived using the logistic regression formulae in two steps. First, ORs were given as exponentials of logistic regression parameters ${ }^{16}$; second, $\mathrm{RDs}$ were derived as a simple function of the ORs considering the maximum Manski bounds for the RDs in classical case-control studies. ${ }^{17}$

Results are given within RLS $\times$ PS strata. Robust (sandwich) $95 \%$ CIs were computed, and $\mathrm{p}<0.05$ on a two-sided test was considered significant. Statistical analyses were performed with SPSS (V.16), and Mplus (V.6.1) packages.

\section{RESULTS}

This study targeted 588 patients with CS among the 1017 enrolled in the IPSYS registry (57.8\%) and 585 control subjects. The causes of stroke in the remaining 429 patients were distributed as follows: large-vessel atherosclerosis and smallvessel disease in $112(11.0 \%)$ and $55(5.4 \%)$ cases, respectively, non-atherosclerotic vasculopathy in 179 (17.6\%) cases, probable cardiac embolism in $22(2.1 \%)$ cases, and other aetiologies in the remaining $61(5.9 \%)$ cases. Demographic characteristics and distribution of risk factors in patients with CS and control subjects are summarised in table 1 . Patients with CS more often had hypertension and hypercholesterolaemia, were more often smokers, and, overall, were more likely to have an unfavourable vascular risk profile than control subjects. RLS was more common in the group of patients $(n=279 ; 47.5 \%)$ than in the group of control subjects ( $n=119 ; 20.3 \%)$.

As summarised in table 2, the 'pure' risk of CS increased with increasing number of proatherosclerotic risk factors in subjects without RLS (OR 2.73; 95\% CI 1.98 to 3.76 ; RD +0.246; 95\% CI +0.17 to +0.32 ; for subjects with one or more factor), but was higher in subjects with RLS and no additional proatherosclerotic factors (PS 0; OR 5.14; 95\% CI 3.49 to 7.58 ; RD +0.388; 95\% CI +0.31 to +0.47$)$ when compared with subjects without RLS and without risk factors. A significant negative interaction and an antagonistic effect between RLS and proatherosclerotic factors were observed. The increase in CS risk with increasing number of proatherosclerotic factors was smaller in subjects with RLS than in subjects without RLS (interaction OR 0.526; 95\% CI 0.31 to 0.91 ; interaction $\mathrm{RD}-0.172 ; 95 \% \mathrm{CI}-0.29$ to -0.05 ; figure 1). In other words, the influence of RLS on the risk of CS was higher when atherosclerotic factors were absent (PS, 0) and reduced (if anything) with an increasing number of these factors (PS $\geq 1$ ).

\section{DISCUSSION}

Despite recent progress in elucidation of the mechanisms linking RLS and ischaemic stroke, identification of patients whose interatrial abnormality is likely to play a pathogenic role is still a matter of debate. It is estimated that at least one-third of RLSs discovered in patients with CS are incidental findings and are unrelated to stroke. ${ }^{18}$ The prevailing idea is that stroke patients who carry an RLS should be subgrouped on the basis of the individual likelihood that the index event is attributable to this anatomical variant and that the presence/absence of coexistent factors thought to modulate the risk of disease may be used to make such an estimation. ${ }^{4} 18$ The results of our study provide evidence that the associated proatherosclerotic conditions is one of these factors, the risk of paradoxical embolisation being increased in subjects with a lower prevalence of conventional stroke risk factors and even higher when they are absent. The increasing burden of these factors had a smaller effect on the risk of CS in patients with RLS than in patients with no evidence of interatrial abnormality. These findings support the notion that different pathogenic mechanisms are operating in the occurrence of cerebral ischaemia in young adults with RLS and that not all
Figure 1 Trajectory of cryptogenic stroke risk presented as risk difference (A) and $O R(B)$ according to right-to-left shunt (RLS) status and proatherosclerotic score.
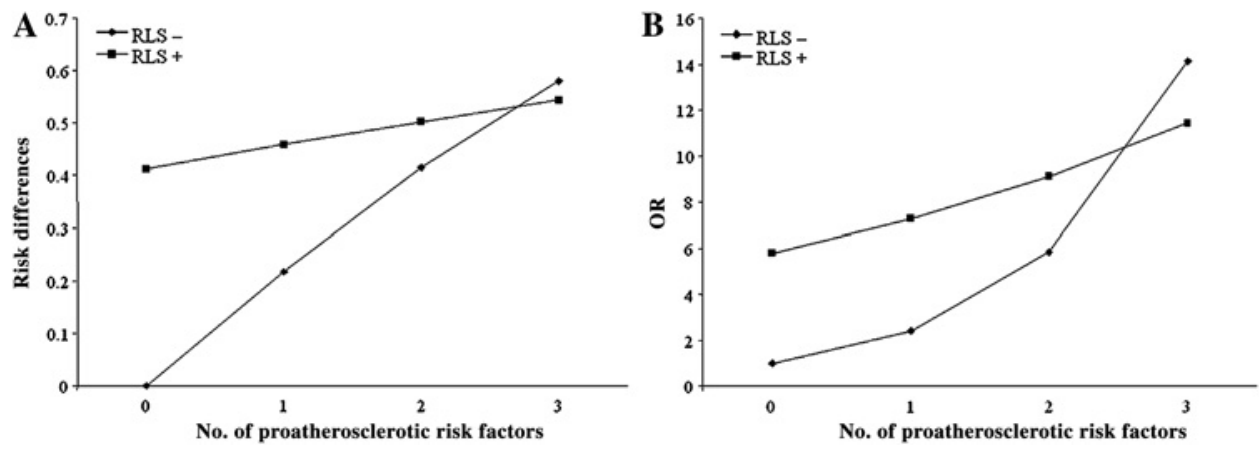
the detected RLSs have a pathophysiological relationship to the index stroke. The practical implication of these findings is noteworthy. Stratifying patients with RLS and otherwise CS into subgroups of higher and lower RLS-attributable risk based on individual proatherosclerotic profiles is a simple and effective way to help reduce patient heterogeneity, allowing more accurate selection of those with probable paradoxical embolism for future therapeutic trials and leading to more targeted treatment approaches. ${ }^{18}$ Our study adds to the accumulating evidence of a lower prevalence of conventional stroke risk factors among CS patients with $\mathrm{PFO}$ than among those without $\mathrm{PFO},{ }^{5-10}$ although the different setting and design of the previous studies and the lack of a group of control subjects make their results not entirely comparable to ours. Also, besides the obvious implication in the process of $\mathrm{PFO}$-carrier stratification, these findings indirectly reinforce the notion that cardiac RLS probably has a role in the occurrence of brain ischaemia.

\section{STRENGTHS AND LIMITATIONS OF THE STUDY}

The study was based on a large and representative cohort with a narrow age range, comprehensive data on conventional risk factors for cerebral ischaemic disease, accurate assessment of RLS, reliable ascertainment of stroke aetiology, and the use of a group of stroke-free control subjects whose RLS status was systematically determined. Another strength of our study was the use of a logistic modelling approach to estimate both multiplicative and additive interaction effects, instead of the simple regression methods that are typically used in $\mathrm{PFO}$-stroke epidemiological studies and are often inadequate for estimating causal effects when the causal structures of interest are complex. This enabled us to look in detail at interactions, allowing a detailed comparison of risk factor profiles between PFO carriers who had a stroke and those who did not, and adds to the generalisability of our results.

Several considerations and limitations may affect the interpretation of our findings. The c-TCD technique prevents the assessment of atrial septal aneurysm-bulging of the interatrial wall sometimes associated with RLS-which has been thought to increase the risk of brain embolism. Nor could we estimate the influence that RLS diameter-a marker that may allow identification of patients at high risk of cerebral ischaemia-may have on the results. This limits interpretation of the data on the strength of the relation between RLS and proatherosclerotic factors on CS occurrence. Also, since the group of controls was recruited among hospital employees, we cannot theoretically rule out the possibility of biased case-control matching, as a consequence of the presumed healthier lifestyle of these subjects. However, since the prevalence of risk factors, as well as of RLS, in our subgroup of controls is similar to that found in other groups of healthy individuals from the same geographical area, ${ }^{19} 20$ we believe such a potential bias did not play a part in our analysis. Finally, because of the young age of our cohort, generalising these findings to other age groups in which cardiac RLS is thought to be pathogenic ${ }^{21}$ should be performed with caution.

\section{CONCLUSION}

In conclusion, the results of our analysis provide evidence of a significant difference in atherosclerotic risk factor profile according to the presence of interatrial RLS in patients with CS and a differential influence of such a cardiac abnormality on the risk of CS according to the number of these factors. Although a causal relationship between RLS and CS remains difficult to ascertain at an individual level, these findings may be useful for the identification of patients whose RLS is likely to be pathogenic.

\section{Author affiliations}

${ }^{1}$ Dipartimento di Scienze Mediche e Chirurgiche, Clinica Neurologica, Università degli Studi di Brescia, Brescia, Italia

${ }^{2}$ Dipartimento di Scienze Sanitarie Applicate, Sezione di Statistica Medica e Epidemiologia, Università di Pavia, Pavia, Italia

${ }^{3}$ Centro Trombosi, IRCCS Istituto Clinico Humanitas, Rozzano-Milano, Italia

${ }^{4}$ Stroke Unit, Azienda Ospedaliera Sant'Andrea, Roma, Italia

${ }^{5}$ Dipartimento di Neuroscienze, Oftalmologia e Genetica, Università di Genova, Genova, Italia

${ }^{6}$ Stroke Unit, Clinica Neurologica, Nuovo Ospedale Civile 'S. Agostino Estense', AUSL Modena, Italia

${ }^{7}$ Dipartimento di Neuroscienze, Scienze Psichiatriche e Anestesiologiche, UOC Neurologia, Università di Messina, Messina, Italia

${ }^{8}$ Istituto di Ricovero e Cura a Carattere Scientifico, Centro Neurolesi Bonino-Pulejo, Messina, Italia

${ }^{9}$ UO Neurologia, Azienda Ospedaliera-Universitaria Borgo Trento, Verona, Italia

${ }^{10}$ Stroke Center, Dipartimento di Neurologia, Ospedale Sacro Cuore Negrar, Verona, Italia

${ }^{11}$ Unità di Neurologia, Ospedale di Circolo, Università dell'Insubria, Varese, Italia

${ }^{12}$ Unità di Neurologia, Ospedale S. Andrea, La Spezia, Italia

${ }^{13}$ Dipartimento di Neuroscienze, Stroke Unit, Università di Torino, Torino, Italia

${ }^{14}$ Stroke Unit, Neurologia Vascolare, Spedali Civili di Brescia, Brescia, Italia

${ }^{15}$ U.O.C. Neurologia, A.O Salerno, Salerno, Italia

${ }^{16}$ Stroke Unit, Divisione di Medicina Cardiovascolare, Università di Perugia, Perugia, Italia

${ }^{17}$ U.0. Neurologia, Istituto Clinico Città di Brescia, Brescia, Italia

${ }^{18}$ Laboratori di Ricerca, Centro di Ricerca e Formazione ad Alta Tecnologia nelle

Scienze Biomediche, 'Giovanni Paolo II', Università Cattolica del Sacro Cuore,

Campobasso, Italia

Acknowledgements We express our gratitude to all the doctors who assisted in the ascertainment and recruitment of patients in the IPSYS centres and to all the individuals who participated in the study.

Contributors AP collected study data, designed and conceptualised the study, analysed and interpreted all data, and drafted the manuscript. MG analysed and interpreted all data, performed statistical analysis, and revised the manuscript. CL, RP, CG, AZ, RM, RSC, PB, AA, MLD, EDelZ, LLR, MR, MDelS, AS, AG, IV, FC, PC, PC, MM, AT, MP, GDV, interpreted and collected study data, and revised the manuscript. LI interpreted study data and revised the manuscript. AP contributed to study design, interpreted study data and revised the manuscript.

\section{Competing interests None.}

Patient consent Obtained.

Ethics approval The study was conducted with the approval of the local ethics committee.

Provenance and peer review Not commissioned; externally peer reviewed.

\section{REFERENCES}

1. Overell JR, Bone I, Lees KR. Interatrial septal abnormalities and stroke: a metaanalysis of case- control studies. Neurology 2000;55:1172-9.

2. Meissner I, Khandheria BK, Heit JA, et al. Patent foramen ovale: innocent or guilty? Evidence from a prospective population-based study. J Am Coll Cardiol 2006;47:440-5.

3. Di Tullio MR, Sacco RL, Sciacca RR, et al. Patent foramen ovale and the risk of ischemic stroke in a multiethnic population. J Am Coll Cardiol 2007;49:797-802.

4. Alsheikh-Ali AA, Thaler DE, Kent DM. Patent foramen ovale in cryptogenic stroke: incidental or pathogenic? Stroke 2009;40:2349-55.

5. Mas JL, Arquizan C, Lamy C, et al; Patent Foramen Ovale and Atrial Septal Aneurysm Study Group. Recurrent cerebrovascular events associated with patent foramen ovale, atrial septal aneurysm, or both. N Engl J Med 2001:345:1740-6.

6. Rodes-Cabau J, Noel M, Marrero A, et al. Atherosclerotic burden findings in young cryptogenic stroke patients with and without a patent foramen ovale. Stroke 2009:40:419-25

7. Lamy C, Giannesini C, Zuber $\mathrm{M}$, et al. Clinical and imaging findings in cryptogenic stroke patients with and without patent foramen ovale. The PFO-ASA study. Stroke 2002;33:706-11.

8. Weimar C, Holle DN, Benemann J, et al; German Stroke Study Collaboration. Current management and risk of recurrent stroke in cerebrovascular patients with right-to-left cardiac shunt. Cerebrovasc Dis 2009;28:349-56.

9. Serena J, Marti-Fàbregas J, Santamarina E, et al; CODICIA, right-to-left shunt in cryptogenic stroke study; stroke Project of the cerebrovascular diseases study group 
Spanish Society of Neurology. Recurrent stroke and massive right-to-left shunt: results from the prospective Spanish multicenter (CODICIA) study. Stroke 2008;39:3131-6.

10. Homma S, Sacco RL, Tullio Di, et al. Effect of medical treatment in stroke patients with patent foramen ovale. Patent foramen ovale in cryptogenic stroke study. Circulation 2002;105:2625-31.

11. Pezzini A, Grassi M, Lodigiani $\mathrm{C}$, et al; Italian Project on stroke in young adults Investigators. Predictors of migraine subtypes in young adults with ischemic stroke: the Italian Project on stroke in young adults. Stroke 2011:42:17-21.

12. Webster MW, Chancellor AM, Smith HJ, et al. Patent foramen ovale in young stroke patients. Lancet 1988;2:11-12.

13. Jauss M, Zanette E. Detection of right-to-left shunt with ultrasound contrast agent and transcranial Doppler sonography. Cerebrovasc Dis 2000;10:490-6.

14. Job FP, Ringelstein EB, Grafen $Y$, et al. Comparison of transcranial contrast Doppler sonography and transesophageal contrast echocardiography for the detection of patent foramen ovale in young stroke patients. Am J Cardiol 1994;74:381-4.
15. Johnson CJ, Kittner SJ, McCarter RJ, et al. Interrater reliability of an etiologic classification of ischemic stroke. Stroke 1995;26:46-51.

16. Cortina-Borja $\mathbf{M}, \mathbf{S m i t h} \mathrm{AD}$, Combarros 0 , et al. The synergy factor: a statistic to measure interactions in complex diseases. BMC Res Notes 2009;2:105.

17. King G, Zeng L. Estimating risk and rate levels, ratios and differences in case-control studies. Statist Med 2002;21:1409-27.

18. Thaler DE, Kent DM. Rethinking trial strategies for stroke and patent foramen ovale. Curr Opin Neurol 2010;23:73-8.

19. Groppo E, De Gennaro R, Granieri G, et al. Incidence and prognosis of stroke in young adults: a population-based study in Ferrara, Italy. Neurol Sci. Published Online First: 22 June 2011. doi:10.1007/s10072-011-0654-9.

20. Patella R, Spalloni A, Ferrari M, et al. Cerebral ischemia in young patients (under 45 years of age): clinical and neuroradiological follow-up. Neurol Sci 2011:32:427-32.

21. Handke M, Harloff $A$, Olschewski M, et al. Patent foramen ovale and cryptogenic stroke in older patients. N Engl J Med 2007;357:2262-8.

\section{Heart online}

Visit Heart online for free editor's choice articles, online archive, email alerts, blogs or to submit your paper. Keep informed and up to date by registering for electronic table of contents at heart.bmj.com. 


\section{HEART}

\section{Interaction between proatherosclerotic factors and right-to-left shunt on the risk of cryptogenic stroke: the Italian Project on Stroke in Young Adults}

Alessandro Pezzini, Mario Grassi, Corrado Lodigiani, et al.

Heart 2012 98: 485-489 originally published online January 23, 2012 doi: 10.1136/heartjnl-2011-300970

Updated information and services can be found at:

http://heart.bmj.com/content/98/6/485.full.html

\section{These include:}

Data Supplement

References

Email alerting service
"Supplementary Data"

http://heart.bmj.com/content/suppl/2012/01/23/heartjnl-2011-300970.DC1.html

This article cites 20 articles, 10 of which can be accessed free at: http://heart.bmj.com/content/98/6/485.full.html\#ref-list-1

Article cited in:

http://heart.bmj.com/content/98/6/485.full.html\#related-urls

Receive free email alerts when new articles cite this article. Sign up in the box at the top right corner of the online article.

Topic Articles on similar topics can be found in the following collections Collections

Notes

To request permissions go to:

http://group.bmj.com/group/rights-licensing/permissions

To order reprints go to:

http://journals.bmj.com/cgi/reprintform

To subscribe to BMJ go to:

http://group.bmj.com/subscribe/ 